\title{
Numerical simulation and analysis of single grain YBCO processed from graded precursor powders
}

J Zou, M D Ainslie, D Hu, W Zhai, N Devendra Kumar, J H Durrell, Y-H Shi, D A Cardwell

Bulk Superconductivity Group, Department of Engineering, University of Cambridge, Trumpington Street, Cambridge, CB2 1PZ, UK

Email: iz351@cam.ac.uk

\begin{abstract}
Large single-grain bulk high-temperature superconducting (HTS) materials can trap high magnetic fields in comparison with conventional permanent magnets, making them ideal candidates to develop more compact and efficient devices, such as actuators, magnetic levitation systems, flywheel energy storage systems and electric machines. However, macro-segregation of Y-211 inclusions in melt processed YBCO limits the macroscopic critical current density $\mathrm{J}_{c}$ of such bulk superconductors, and hence, the potential trapped field. Recently, a new fabrication technique with graded precursor powders has been developed, which results in a more uniform distribution of $Y$ 211 particles, in order to further improve the superconducting properties of such materials. In order to develop this graded fabrication technique further, a 3D finite-element numerical simulation based on the $\boldsymbol{H}$-formulation is performed in this paper. The trapped field characteristics of a graded YBCO sample magnetized by the field cooling (FC) method are simulated to validate the model, and the simulation results are consistent with the experimental measurements. In addition, the influence of the graded technique and various graded $J_{c}$ distributions for pulsed field magnetization (PFM), recognised widely as a practical route for magnetising samples in bulk superconductor applications, is also investigated, with respect to the trapped field and temperature profiles of graded samples. This modelling framework provides a new technique for assessing the performance of various sizes and geometries of graded bulk superconductors, and by adjusting the $\mathrm{Y}-211$, and hence $\mathrm{J}_{c}$, distribution, samples can be fabricated based on this concept to provide application-specific trapped field profiles, such as the generation of either a high magnetic field gradient or a high level of uniformity for the traditionally conical, trapped field profile.
\end{abstract}

\section{Introduction}

Large, single-grain high-temperature superconducting (HTS) materials such as Y-Ba-Cu-O (YBCO) have considerable potential to trap large magnetic fields over $17 \mathrm{~T}$ at temperatures below $30 \mathrm{~K}$ [1], [2] and up to $3 \mathrm{~T}$ at the technologically important temperature of $77 \mathrm{~K}$ [3]. There have been numerous demonstrations over recent years of HTS materials fabricated into disc shapes replacing permanent magnets in practical applications such as rotating machines [4] and magnetic bearings [58]. The magnitude of trapped field in YBCO samples is determined primarily by their ability to maintain high critical current densities $\left(J_{c}\right)$ even in the presence of large applied magnetic fields [9], enabling large shielding supercurrents to flow over relatively large areas within well-aligned grains of the material [10]. The development of the so-called top-seeded melt-growth (TSMG) technique overcomes, to a large extent, the limitation of $\mathrm{J}_{\mathrm{c}}$ associated with the presence of grain boundaries 
(or weak links) in the sample microstructure. Furthermore, through the introduction of effective pinning centres, flux pinning forces, which are essential to achieving high $J_{c}$, have also been enhanced in these materials. The TSMG technique enables fabrication of large, single grain YBCO bulk samples with good grain alignment and orientation, resulting characteristically in high $\mathrm{J}_{\mathrm{c}}[11]$. It is necessary to engineer a homogeneous distribution of small sized $\mathrm{Y}_{2} \mathrm{BaCuO}_{5}(\mathrm{Y}-211)$ inclusions within the superconducting bulk $\mathrm{YBa}_{2} \mathrm{Cu}_{3} \mathrm{O}_{7-\delta}(\mathrm{Y}-123)$ microstructure during the TSMG growth process in order to fabricate single grain $\mathrm{YBCO}$ samples with improved superconducting properties [12-14]. However, macro-segregation of Y-211 inclusions in melt processed $Y B C O$ has been observed by several groups [15-19] to result in non-uniformity in the distribution of $J_{c}$ across the sample, which is detrimental to the field trapping capability of these materials [20].

A fabrication technique based on grading the Y-211 phase content in the green precursor body has been developed recently by the Cambridge Bulk Superconductivity Group [20]. Here, three types of precursor powders containing different concentrations of Y-211 are arranged into U-shaped stacks within a bulk YBCO precursor pellet. The aim of the graded TSMG processing technique is to manufacture single grain YBCO bulk samples with a more uniform Y-211 particle distribution and associated improved superconducting properties. This is expected to improve homogeneity throughout the bulk, including locations from the centre to the edge, and top to bottom, as well as around the ab-plane, which has a number of merits, as will be described hereafter.

The field trapping capability of the samples fabricated by the graded TSMG technique needs further development compared to those of standard, TSMG processed YBCO samples, even though the grading technique yields a remarkable improvement in the homogeneity of the distribution of Y-211 phase in the bulk microstructure. This paper employs a 3D finite-element model to analyze the properties of YBCO samples fabricated with various $J_{c}$ distributions based on varying the weight percentage content of $\mathrm{Y}-211$ particles to promote and widen the range of potential applications of graded superconductors and to optimize the superconducting properties of the samples to enable the development of an optimized fabrication process. The model used is based on the $\boldsymbol{H}$-formulation [21-31] and implemented in COMSOL Multiphysics 4.3a [32], which is an appropriate platform for investigating the electromagnetic properties of such samples. The key critical current density parameters have been determined experimentally from magnetic moment hysteresis loops for small samples as a function of position within the sample and these are used to provide inputs to the model. The field trapping predictions of the 3D model are compared with the results of the experiment and observed to agree well. Finally, the 3D model is used to investigate the performance of the graded samples containing different distributions of $J_{c}$ that have been magnetized by field cooling (FC) and pulsed field magnetisation (PFM) techniques. Overall, the 3D simulation provides an insight into the properties of the graded material for the development of potential applications.

\section{Sample fabrication and characterisation}

\subsection{Sample fabrication}

A graded, single grain YBCO sample was fabricated using the top seeded melt growth (TSMG) process [20]. Three layers of precursor powders were arranged with varying Y-211 content from the centre to the edges and bottom of the pellet for the graded sample. The resulting U-shaped arrangement of the cylindrical stack was such that the Y-211: Y-123 weight ratio increased both in 
the radial and axial directions from the centre of the pellet to both its edge and its bottom. For this purpose, precursor powders containing $40 \mathrm{wt} \%$ of $\mathrm{Y}-211$ were used for the region close to the vicinity of the seed, decreasing to $30 \mathrm{wt} \%$ in the middle and $20 \mathrm{wt} \%$ towards the outer $\mathrm{U}$ layer of the pellet. The top, middle and bottom regions in graded sample are denoted as $T, M$ and $B$, respectively, and this is shown schematically in Figure 1 (a).

The sample was fabricated from an equal volume and weight of precursor powders, and was approximately $25 \mathrm{~mm}$ in diameter and $14 \mathrm{~mm}$ in height after melt processing.

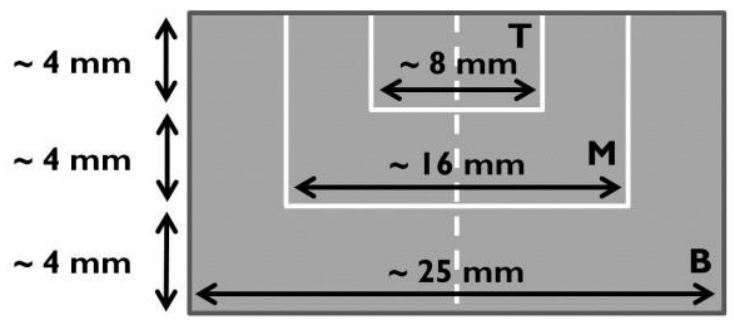

(a)

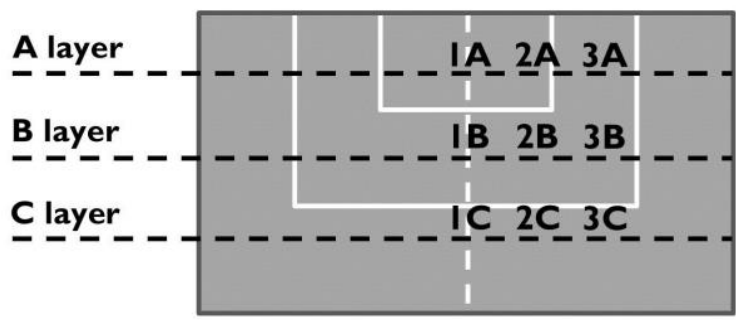

(b)

Figure 1. Graded sample geometry: (a) schematic diagram of a graded bulk YBCO sample fabricated by varying the weight of $\mathrm{Y}-211$ in the precursor powders $(\mathrm{T}=40 \mathrm{wt} \%, \mathrm{M}=30 \mathrm{wt} \%$ and $\mathrm{B}=$ $20 \mathrm{wt} \%$, from the centre to the edge/bottom of the pellet) arranged in a nested, Ushaped cylindrical stack and (b) positions of the small specimens within the parent bulk cut from each layer for measurement of $J_{c}$ for input to the model [20].

\subsection{Trapped field measurements}

An existing scanning system consisting of a linear array of Hall probes was used to measure the trapped field of the sample fabricated in the current study [33]. The sample was field cooled (FC) in an external magnetic field of $1.5 \mathrm{~T}$ from room temperature to $77 \mathrm{~K}$. The Hall probe array was positioned approximately $0.5 \mathrm{~mm}$ above the sample during this measurement. The experimentally measured trapped field profile is compared with the modelling results in Section 3.2. The sample was cut into three layers, labelled as A, B and C representing the top, middle and bottom sections, as shown in Figure 1(b), in order to investigate the performance of the samples as a function of their thickness. Each of these layers was approximately $3 \mathrm{~mm}$ thick, as indicated by the dashed lines in the figure.

\section{$2.3 \mathrm{~J}_{\mathrm{c}}$ characteristics and estimation}

Small sub-specimens of dimensions approximately $1.5 \mathrm{~mm} \times 2.0 \mathrm{~mm} \times 1.2 \mathrm{~mm}$ were cut from each of the three layers A, B and C for measurement of their critical current density $\left(J_{c}\right)$. Magnetic moment hysteresis $(\mathrm{m}-\mathrm{H})$ loops were measured at $77 \mathrm{~K}$ from each sub-specimen using a Quantum Design Magnetic Property Measurement System (MPMS) XL SQUID magnetometer by applying the field parallel to the c-axis ( $\mathrm{H}|| \mathrm{c}$-axis) of the sample. The variation of critical current density with field was determined for each sample using the extended Bean model [34].

To model the electromagnetic behaviour of bulk HTS materials, a macroscopic approximation is usually made based on some local measurements of small samples, for example [35], followed by 
application of a Kim or Kim-like model [36]. Kim showed in [36] that 'hard,' or type II, superconductors, like HTS materials, exhibit $J_{c}$ behaviour that follows the following empirical relationship similar to that given in equation (1). In this paper, the following modified Kim-like model, which has been applied to bulk HTS material previously modelling in [29, 37-40], is employed to estimate $J_{c}(B)$ data for each sample as the input data for the 3D model:

$$
J_{c}(B)=\frac{J_{c 0}(T)}{\left(1+\frac{B}{B_{0}}\right)^{\beta}}
$$

where $J_{c}$ is the local critical current density, $B$ is the magnitude of the local field, $B_{0}$ and $\beta$ are material-dependent constants and $J_{c 0}$ is the magnitude of critical current density when the local field is zero.

Due to the normally inhomogeneous $J_{c}$ distribution formed in such materials [41, 42], knowing the $J_{c}$ distribution in terms of different position within the sample is very important and there do not yet exist any non-destructive methods to evaluate this.

The values of $\mathrm{J}_{\mathrm{c} 0}$ and $\mathrm{B}_{0}$ for the three $\mathrm{U}$-shaped regions in the graded bulk sample are quite different due to the varying $\mathrm{Y}-211$ content and distribution. Here, the parameters within the top U-shaped section are labelled as $\mathrm{J}_{\mathrm{c} 1}$ and $\mathrm{B}_{01}$, with the corresponding parameters in the middle and bottom $\mathrm{U}$ shaped geometry labelled as $\mathrm{I}_{\mathrm{c} 2}, \mathrm{~B}_{02}$ and $\mathrm{J}_{\mathrm{c} 3}, \mathrm{~B}_{03}$, respectively.

In light of these considerations, taking several specimens throughout the bulk (in this paper, nine specimens are considered) and taking an average $J_{c}$ for each U-shape region in the bulk can help us to predict the macroscopic behaviour of the bulk [35]. The modified critical current densities were computed for each of the three U-shaped regions as follows, shown in Figure 2. Essentially, the more small samples used to map the $J_{c}$ distribution of (RE)BCO samples, the better the input data is for the modelling work and the more representative of the macroscopic superconductor properties.

$$
\begin{gathered}
J_{c 1}=\left(J_{C 1 A} J_{c 2 A}\right) / 2 \\
J_{c 2}=\left(J_{c 1 B}+J_{c 2 B}+J_{C 3 B}+J_{C 3 A}\right) / 4 \\
J_{C 3}=\left(J_{c 1 C+} J_{c 2 C}+J_{c 3 C}\right) / 3
\end{gathered}
$$

The key parameters of the critical current densitiesfor the graded YBCO sample was determined using a MATLAB fit and are shown in Table 1. The data fitting over from the range of 0-2 T are shown in Figure 2, in which the open symbols represent the experimental data and the solid lines represent the results from the data fitting. It can be seen that the critical current density at zero field of the graded sample increases from the seed towards the bottom of the sample, which is consistent with data reported previously [20]. A spatial change in the concentration of Y-211 inclusions from high at the centre of the bulk, to low at the bottom, compensates the macro-segregation of Y-211 particles in the graded sample. 
Table 1. Parameters calculated by data fitting using MATLAB

\begin{tabular}{cc}
\hline PARAMETER & GRADED SAMPLE \\
\hline $\mathrm{J}_{\mathrm{c} 01}$ & $2.45 \times 10^{8} \mathrm{~A} / \mathrm{m}^{2}$ \\
$\mathrm{~J}_{\mathrm{c02}}$ & $3.62 \times 10^{8} \mathrm{~A} / \mathrm{m}^{2}$ \\
$\mathrm{~J}_{\mathrm{c} 03}$ & $3.96 \times 10^{8} \mathrm{~A} / \mathrm{m}^{2}$ \\
$\mathrm{~B}_{01}$ & $0.1534 \mathrm{~T}$ \\
$\mathrm{~B}_{02}$ & $0.0685 \mathrm{~T}$ \\
$\mathrm{~B}_{03}$ & $0.0780 \mathrm{~T}$ \\
$\beta_{1}$ & 0.5402 \\
$\beta_{2}$ & 0.45 \\
$\beta_{3}$ & 0.585 \\
\hline
\end{tabular}

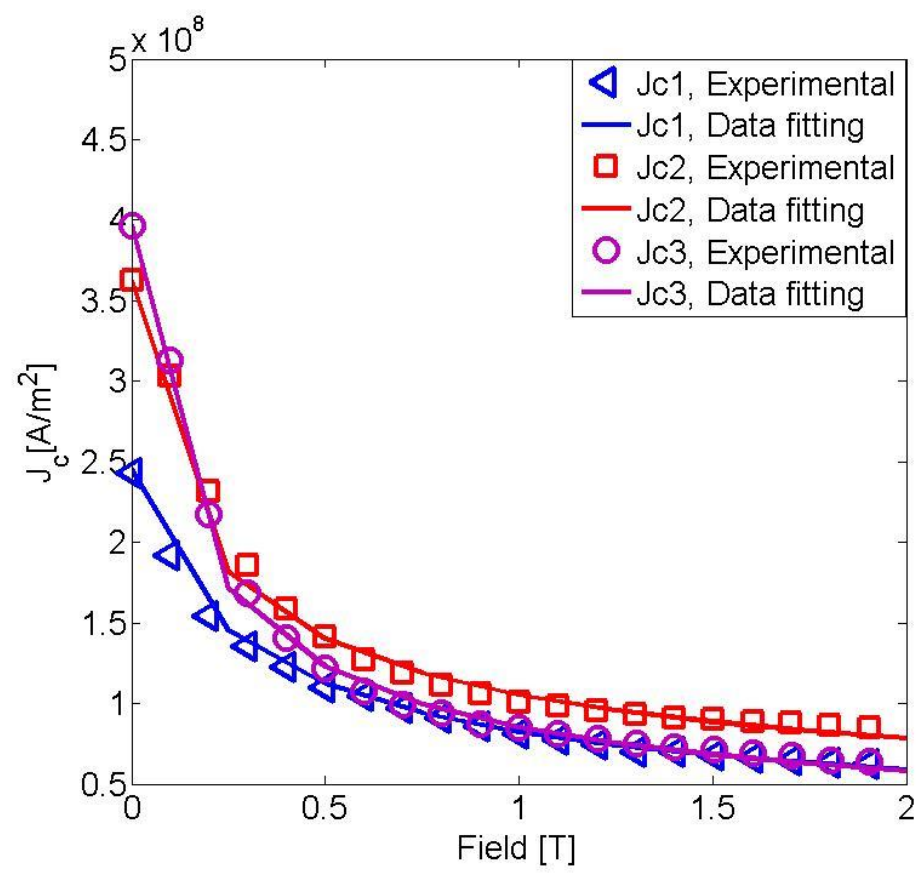

Figure 2. Data fitting based on the experimental data of field dependence, $J_{c}(B)$, of the modified critical current densities at $77 \mathrm{~K}$ of the sub-specimens [20] cut from the graded sample. 


\section{Modelling framework\& verification}

The numerical simulation of superconducting materials is a powerful tool to help explain experimental results, investigate the physical mechanisms underlying the observed behaviour and to predict the performance of HTS materials in general. In addition, in contrast to analytical techniques, numerical simulations can predict the performance of HTS samples with complex geometries and properties, which can provide detailed insight for engineering both materials and devices.

The present section addresses the computation of the electromagnetic properties of the graded and standard samples magnetized by field cooling using the $\boldsymbol{H}$-formulation [21-31], which was simulated using the 3D Partial Differential Equation (PDE) module of the commercial software package COMSOL Multiphysics 4.3 a [32].

In this 3D model, there are three independent variables: $\boldsymbol{H}=\left[H_{x}, H_{y}, H_{z}\right]$. The governing equations are based on Maxwell's equations (Faraday's (5) and Ampere's (6) laws), as follows:

$\nabla \times E=-\frac{\partial B}{\partial t}=-\frac{\partial H}{\partial t}$

$\nabla \times H=J$

In the field-dependent equation (1), B is defined by the following equation:

$$
B=\mu_{0} \sqrt{H_{x}^{2}+H_{y}^{2}+H_{z}^{2}}
$$

Based on a simple Bean model [46] approximation, as long as the field applied fully penetrates the bulk, in FC the bulk traps the field applied. In ZFC, if a field at least twice this full penetration field is applied; the trapped field will be same as in the case of FC [40]. Therefore as long as the bulk is full penetrated in the model, a ZFC process in this simulation can represent the FC process in experiment.

In the FC simulation, the magnitude of the field applied in these modelling examples is set in such a way to fully penetrate the superconductor and ensure the same results. For the simulation of PFM, an existing model that allows multiphysics (both electromagnetic and thermal properties as required for PFM) to be simulated has been extended [47].

The following thermal transient equation is employed in the thermal model:

$$
\rho \cdot C \frac{d T}{d t}=\nabla \cdot(k \nabla T)+Q
$$

Where $\rho$ is the mass density, $\mathrm{C}$ is the specific heat, $\mathrm{Q}$ is the generated heat and $\mathrm{k}$ is the thermal conductivity. The critical current density in zero field, $J_{c 0}$, is then modified as equation (9), in order to couple the electromagnetic and thermal models and provide a temperature-dependent $\mathrm{J}_{\mathrm{c}}[47-49]$.

$$
J_{c 0}(T)=\alpha\left[1-\left(\frac{T}{T_{c}}\right)^{2}\right]^{1.5}
$$


Where $\alpha$ is the critical current density extrapolated to $\mathrm{T}=0 \mathrm{~K}$. The generated heat, $\mathrm{Q}$, is the product of the electric field and current density throughout the superconductor $(Q=E \bullet J)$. The same thermal parameters are used as detailed in [47].

While the 3D model in this particular case is axisymmetric due to the assumptions made regarding the $J_{c}(B, T)$ behaviour, this 3D model is a step towards developing more complicated models in terms of $J_{c}(B, T)$ behaviour, as well as superconductor geometry (square-, hexagonal- and bar-shaped samples, for example), which can be extended in the future. In addition, a 3D model allows application of fields other than perpendicular to the top surface, including studies into the effects of externally applied AC fields on the trapped fields in bulk superconductors.

The electromagnetic behaviour of the superconductor is modelled using the E-J power law [50,51], E $\alpha \mathrm{J}^{\mathrm{n}}$, where $\mathrm{n}=21[23,47,48]$ and $\mathrm{E}_{0}=1 \times 10^{-4} \mathrm{Vm}^{-1}$. For HTS materials, $\mathrm{n}$ usually varies between 5 (strong flux creep) and 50 (limiting value between HTS and LTS materials) [27,52,53], and for $n>20$, it becomes a good approximation of Bean's critical state model [54]. The electromagnetic properties are analysed with each of the three $\mathrm{U}$-shaped regions described by the $\mathrm{J}_{\mathrm{c} 0}, \mathrm{~B}_{0}$ and $\mathrm{n}$ parameters given in equation (1) and shown schematically in Figure 3. In the PFM simulation, the bulk was immerged into liquid nitrogen as shown in Fig 3, which maintains the temperature around $77 \mathrm{~K}$. The current was assumed to flow parallel to the electric field in the present simulation, which is considered both to be a suitable macroscopic assumption for bulk superconductors and keeps open the problem of flux cutting $[55,56]$.

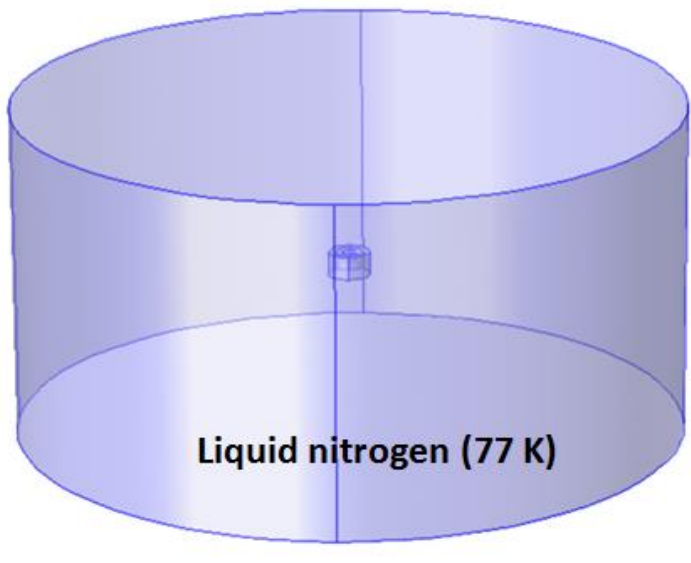

(a)

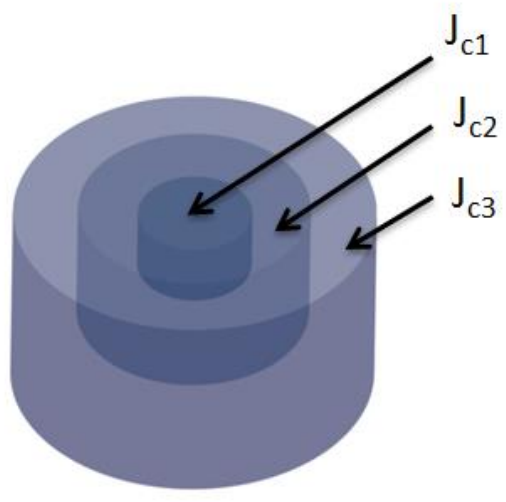

(b)

Figure 3. Sample geometry used in the 3D simulation (a) the complete model (including the model boundary) and (b) the bulk HTS only.

An external magnetic field of magnitude $2 \mathrm{~T}$ was applied to the bulk along the z-axis (corresponding to the c-axis of the bulk) to simulate field cooling of the sample for the trapped field measurements, as shown in Figure 4, by setting appropriate boundary conditions in the model [47]. In this case, the magnitude of the applied field was sufficiently large to ensure full penetration of the bulk sample at $77 \mathrm{~K}$. The trapped field profile was then calculated at $0.5 \mathrm{~mm}$ above the bulk YBCO surface at $\mathrm{t}=25 \mathrm{~s}$, allowing sufficient time for the flux in the sample to relax. 
The simulated trapped field profile for graded sample is shown in Figure 5 and compared with the experimental results presented previously in [20]. The experiments revealed that the FC graded YBCO sample exhibited a maximum trapped field of $0.65 \mathrm{~T}$ at $77 \mathrm{~K}$. The results of the experiment and the model agree reasonably well with these considerations. In the following section, numerical analyses with respect to application-specific trapped fields for different HTS applications are carried out, for which the graded technique is potentially important.

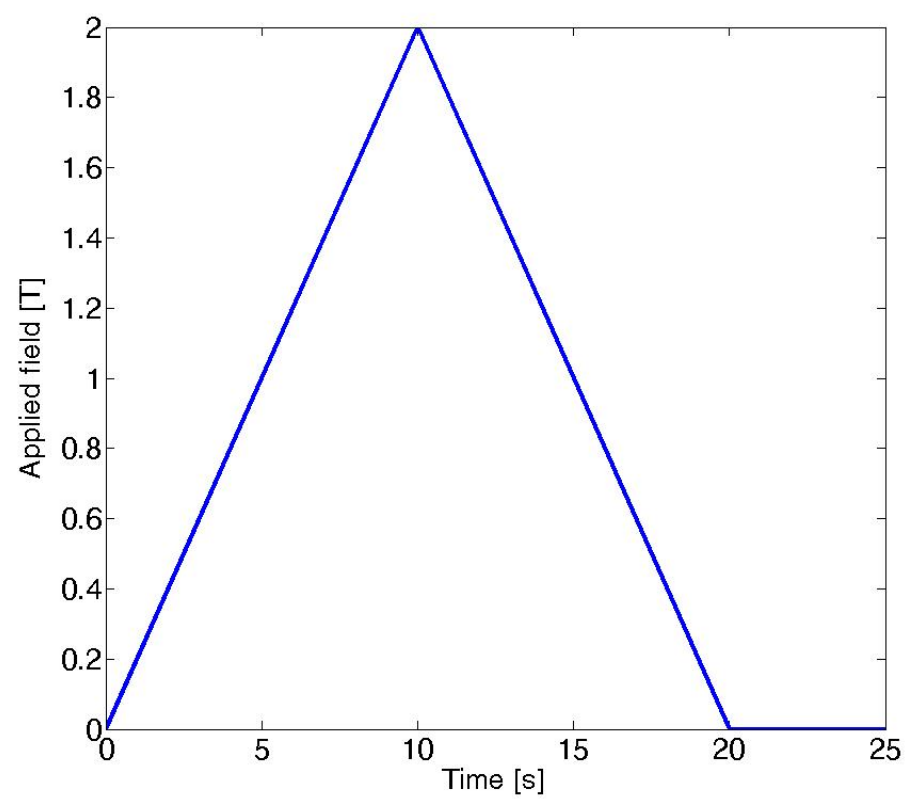

Figure 4. Applied magnetic field for the simulation of field cooling of the YBCO samples.

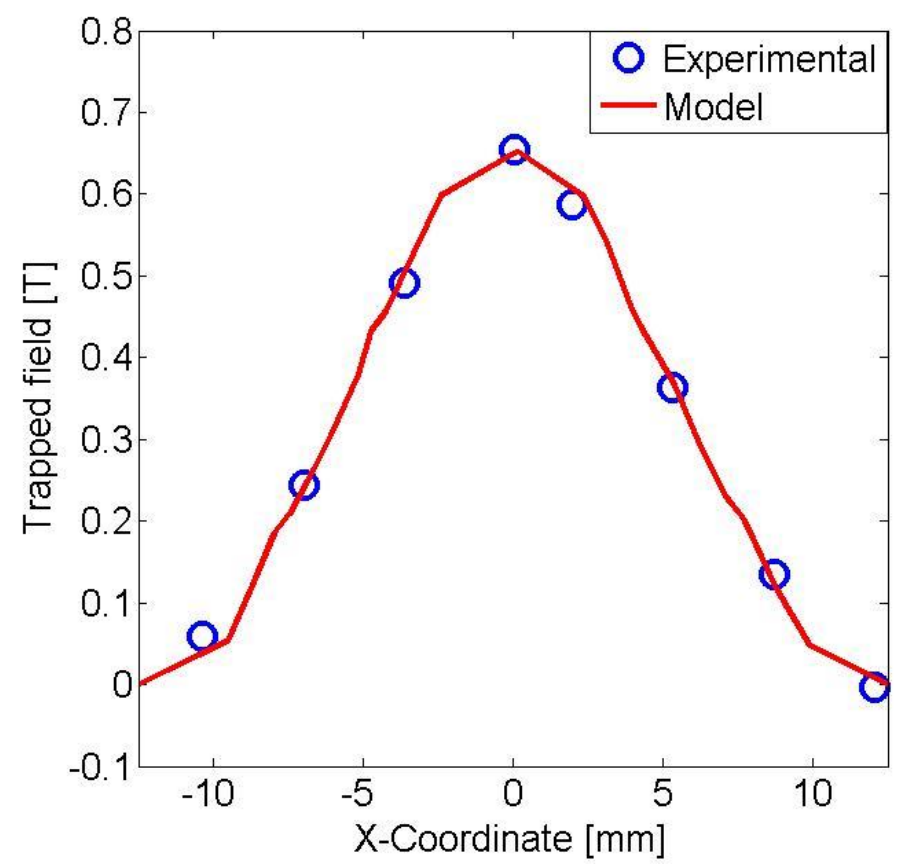

Figure 5. Comparison of the results of the experiment and the model for the graded sample. 


\section{Potential applications}

\subsection{Effect of graded technique on FC}

It can be seen from Table 1 that the maximum $J_{c}$ in the graded bulk sample occurs in the bottom $\mathrm{U}$ shape region of the sample. Due to the redistribution of Y-211 particles, the graded technique can produce a homogeneous $J_{c}$ around the bulk (in the ab-plane), reducing the effect of non-uniform $J_{c}$ between growth sector boundaries (GSBs) and growth sector regions (GSRs) and between the centre and edge, but could also produce different (but still homogeneous around the ab-plane) distributions within each $U$-shaped region to vary the shape of the trapped field profile. The distribution of $\mathrm{Y}-211$ particles in the different U-shaped regions of the graded sample influences significantly the local value of $J_{c}$ value and the simulation of these effects may enable a better understanding of the material fabrication process to be developed.

Five possible profiles (Models A-E) of magnetic field generated from controlled current distributions were investigated as part of this study. All the parameters in the models from $A$ to $E$ are selected based on historical data from standard YBCO samples fabricated by our group with consideration to the values presented in Table 1 . Models $A$ and $E$ are reference models for a completely homogeneous sample, with an average $J_{c}$ of $3.5 \times 10^{8} \mathrm{~A} / \mathrm{m}^{2}$ and $7 \times 10^{8} \mathrm{~A} / \mathrm{m}^{2}$ respectively, which are within the range $5 \times 10^{7}$ to $1 \times 10^{9} \mathrm{~A} / \mathrm{m}^{2}$, that are normal $\mathrm{J}_{\mathrm{c}}$ values fabricated by the Cambridge Bulk Superconductivity Group [43-45]. Models B-D are for a graded profile of Y-211 particles across the sample, which is achievable using the new graded processing technique. The values of $J_{c 0}$ used in each model are listed in Table 2. $B_{0}$ is assumed to be $0.4 \mathrm{~T}$ for Models A-E, which is within the typical range of material properties of (RE)BCO bulk samples [43], with an identical sample geometry assumed in each case.

Table 2 Model Parameters

\begin{tabular}{llll}
\hline Model & $\begin{array}{l}\text { Top U-shaped } \\
\text { region }\left[\mathrm{A} / \mathrm{m}^{2}\right]\end{array}$ & $\begin{array}{l}\text { Middle U-shaped } \\
\text { region }\left[\mathrm{A} / \mathrm{m}^{2}\right]\end{array}$ & $\begin{array}{l}\text { Bottom U-shaped } \\
\text { region }\left[\mathrm{A} / \mathrm{m}^{2}\right]\end{array}$ \\
\hline $\mathrm{A}$ (Homogeneous $\mathrm{J}_{\mathrm{c}}$ distribution) & $\mathrm{J}_{\mathrm{c} 01}=3.5 \times 10^{8}$ & $\mathrm{~J}_{\mathrm{c} 02}=3.5 \times 10^{8}$ & $\mathrm{~J}_{\mathrm{c} 03}=3.5 \times 10^{8}$ \\
$\begin{array}{l}\mathrm{B} \text { (Graded sample, highest } \mathrm{J}_{\mathrm{c}} \text { in the } \\
\text { bottom region) }\end{array}$ & $\mathrm{J}_{\mathrm{c} 01}=3.5 \times 10^{8}$ & $\mathrm{~J}_{\mathrm{c} 02}=3.5 \times 10^{8}$ & $\mathrm{~J}_{\mathrm{c} 03}=7 \times 10^{8}$ \\
$\begin{array}{l}\mathrm{C} \text { (Graded sample, highest } \mathrm{J}_{\mathrm{c}} \text { in the } \\
\text { middle region) }\end{array}$ & $\mathrm{J}_{\mathrm{c} 01}=3.5 \times 10^{8}$ & $\mathrm{~J}_{\mathrm{c} 02}=7 \times 10^{8}$ & $\mathrm{~J}_{\mathrm{c} 03}=3.5 \times 10^{8}$ \\
$\begin{array}{l}\mathrm{D} \text { (Graded sample, highest } \mathrm{J}_{\mathrm{c}} \text { in the } \\
\text { top region) }\end{array}$ & $\mathrm{J}_{\mathrm{c} 01}=7 \times 10^{8}$ & $\mathrm{~J}_{\mathrm{c} 02}=3.5 \times 10^{8}$ & $\mathrm{~J}_{\mathrm{c} 03}=3.5 \times 10^{8}$ \\
$\begin{array}{l}\mathrm{E} \text { (Homogeneous distribution with } \\
\text { relative high } \mathrm{J}_{\mathrm{c}} \text { ) }\end{array}$ & $\mathrm{J}_{\mathrm{c} 01}=7 \times 10^{8}$ & $\mathrm{~J}_{\mathrm{c} 02}=7 \times 10^{8}$ & $\mathrm{~J}_{\mathrm{c} 03}=7 \times 10^{8}$ \\
\hline
\end{tabular}




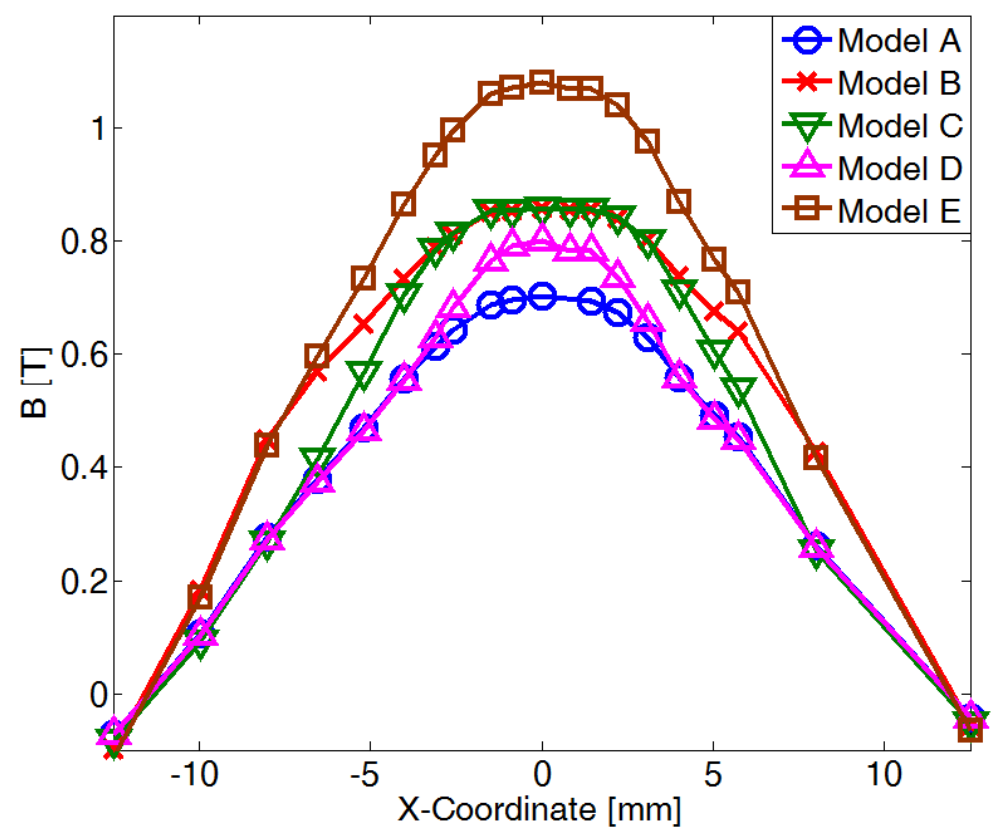

Figure 6. Simulated trapped field profiles for models A-E.

The trapped field profiles on the top surface of the samples for Models A-E are shown in Figure 6. Models $B$ and $C$ have a wider, flatter trapped field profile, as well as higher maximum trapped fields, resulting in higher trapped flux, in comparison with Models $A$ and D. Model $E$ corresponds to an overall improvement of $J_{c}$ across the whole sample, which enlarges the peak value of the trapped field and the maximum trapped flux. From the modelling results, the greatest contribution to the trapped flux is seen in Model $B$, which has a higher $J_{c}$ in the bottom U-shaped region - this is traditionally the region that exhibits the lowest $J_{c}$, which can be addressed using this graded technique.

The shape of the trapped field profile obtained after field cooling for each model is determined by the critical current distribution, which is explained effectively by the Bean model [46]. The local critical current density in the graded bulk samples, therefore, can be varied effectively by varying the distribution of Y-211 particles in the precursor pellet. As a result, the graded technique is a promising method for potentially modifying the trapped field profile to meet the specific requirements of different applications.

\subsection{Effect of graded technique on PFM}

Pulsed field magnetization (PFM) is recognized widely as a practical route for magnetizing samples in bulk superconductor applications [47], since it is of potentially lower cost, requires less time to magnetise the sample and has a smaller magnetization system footprint than FC and zero field cooling (ZFC) techniques. Compared with FC and ZFC, PFM applies an external magnetization field via a pulse of duration typically less than a second, instead of ramping an external field via large coils over minutes or hours. The capability of trapping field by PFM using the graded sample is described in this section. 
The magnitude of the trapped field achieved typically in the PFM process is limited generally by relatively large temperature increases within the bulk sample due to the rapid dynamic movement of magnetic flux lines during the magnetization process [59]. Therefore, the optimal pulsed field for full activation of the graded samples needs to be determined carefully, considering the increased temperature associated with the PFM process. A more homogeneous and improved $\mathrm{J}_{\mathrm{c}}$ distribution has a number of benefits, which are particularly related to the PFM technique. As described in $[38,39,60]$, inhomogeneities in the bulk cause the flux to move preferentially through certain region of the bulk resulting in an inhomogeneous and lower trapped field as well as localised heating within certain regions of the sample.

In this section, the combined 3D electromagnetic-thermal model described in Section 3 is used to model the PFM process for the graded sample. A rise time of $\tau=12 \mathrm{~ms}$ was used for the PFM pulse and the pulse width was approximately $120 \mathrm{~ms}$, which is adequately representative of experimental conditions [47]. The simulation was separated into two time domains, $0<\mathrm{t}<0.12 \mathrm{~s}$ and $0.12 \leq \mathrm{t}<$ $0.3 \mathrm{~s}$, with $\mathrm{n}$ values of 10 and 21 , respectively, as described in [48] to improve convergence of the model.

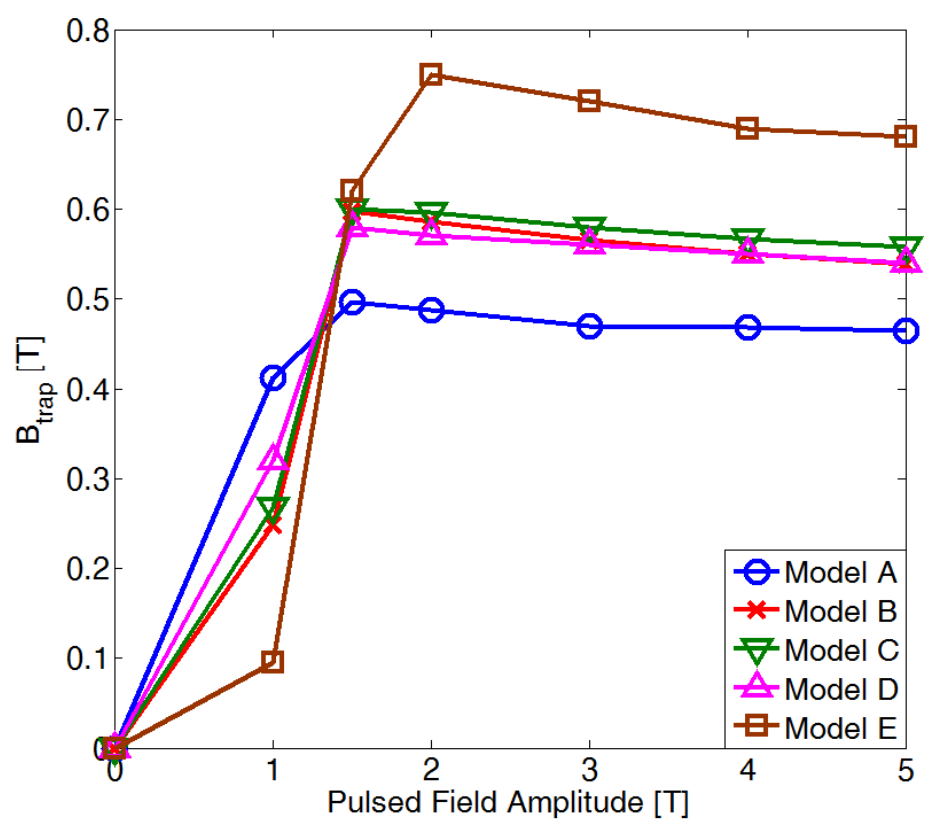

Figure 7. Maximum trapped field at the centre of the bulk superconductor, calculated at the top surface of the sample. Pulsed fields of up to $5 \mathrm{~T}$ were applied at $77 \mathrm{~K}$.

The value of the peak trapped field at the centre of the bulk sample is defined as $B_{\text {trap }}$. The maximum trapped field profiles for the five models at $77 \mathrm{~K}$ for pulsed field amplitudes of up to $5 \mathrm{~T}$ are shown in Figure 7. Each sample has a particular activation field for full magnetization, which is consistent with experimental results reported previously [47]. The trapped field decreases with increasing amplitude of external field above this optimal field due to increasing temperature rise in the bulk sample.

The optimum activation fields for Models A-D are almost the same, around 1.5 T. However, Model E has a relatively larger optimal field, around $2 \mathrm{~T}$, due to its larger overall $\mathrm{J}_{\mathrm{c}}$ values. Models $\mathrm{A}$ and $\mathrm{E}$ show similar trends for applied fields above their optimum activation field, whereby $B_{\text {trap }}$ reduces 
due to an increased temperature rise from flux movement [47]. $B_{\text {trap }}$ in Models $B, C$ and $D$ follow a particular trend, and are higher than that of Model $A$ when the applied field exceeds $2 \mathrm{~T}$. Comparing the graded sample models (Models B, C and D), the measured trapped field is largest in Model C for the PFM process, in contrast to the FC technique, which generates similar trapped fields in Models $\mathrm{B}$ and $\mathrm{C}$.

Figure 8 shows the maximum temperature rise in the ab-plane $2 \mathrm{~mm}$ below the top surface of the sample during PFM for each model, which includes temperature profiles for all three U-shaped regions. Figure 9 compares the two-dimensional temperature distributions for each model at the pulse peak ( $t=12 \mathrm{~ms}$ ) and pulse end $(t=120 \mathrm{~ms}$ ) for an intermediate applied pulsed field $(3 \mathrm{~T})$ in the ab-plane of each sample. The temperature increases most significantly in the region that has the highest $J_{c}$, due to the higher energy dissipated associated with the movement of flux lines against stronger pinning forces. Model B always exhibits the highest temperature rise during the PFM process, which has the highest $J_{c}$ in the outmost cup of the graded sample.

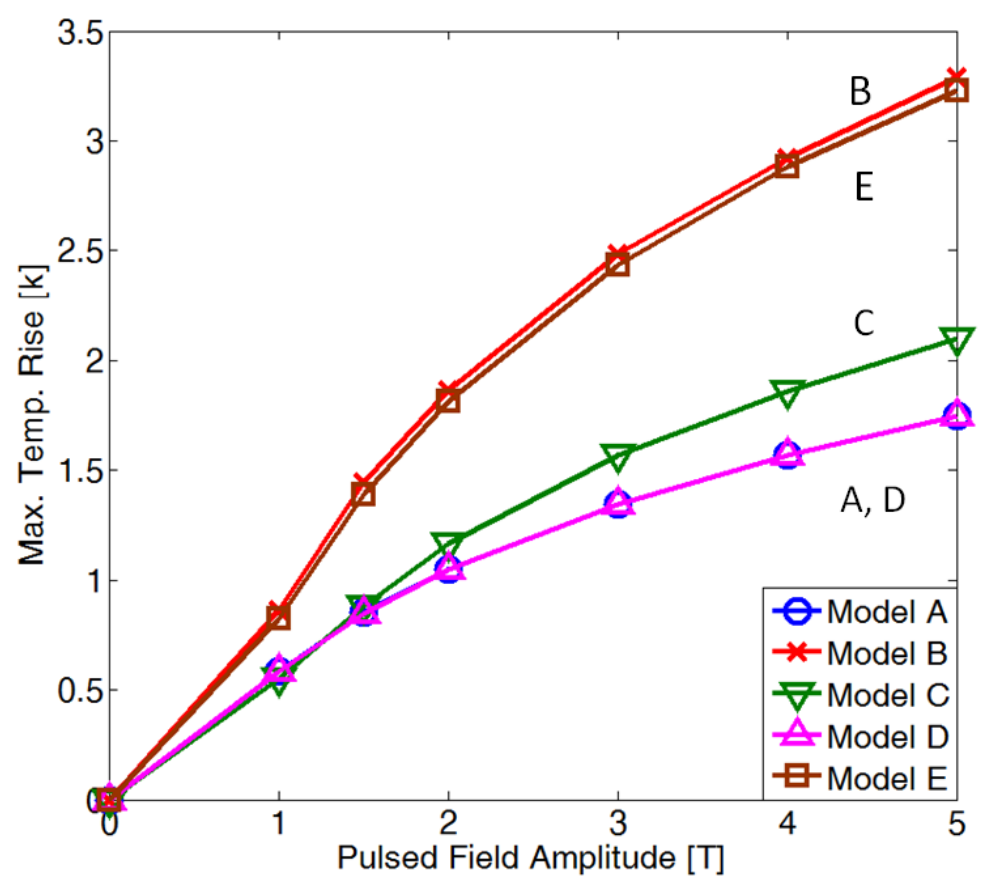

Figure 8. Maximum temperature rise in the ab-plane, $2 \mathrm{~mm}$ below the top surface of the sample, at the pulse peak $(t=12 \mathrm{~ms})$. Pulsed fields of up to $5 \mathrm{~T}$ are applied to the bulk samples at $77 \mathrm{~K}$. 


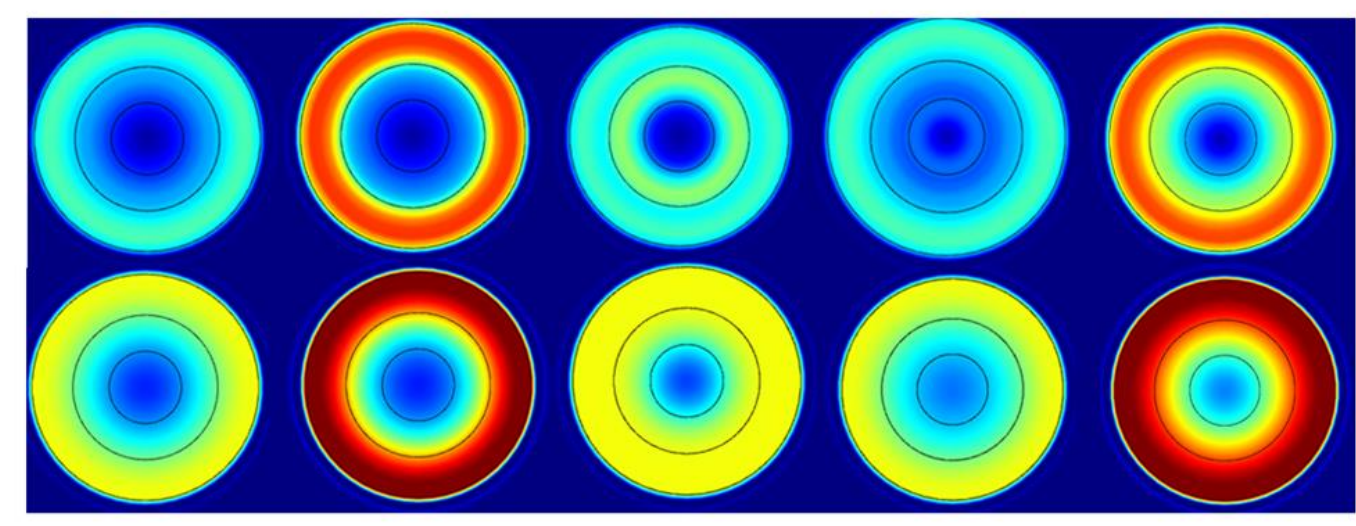

Figure 9. Comparison of the two-dimensional temperature distributions during PFM for Models A-E (from left to right, respectively) at the pulse peak ( $t=12 \mathrm{~ms}$ ) and pulse end ( $t=120 \mathrm{~ms}$ ) for an intermediate applied pulsed field of amplitude 3 T.As in the case of Figure 8 , the results are shown for the ab-plane, $2 \mathrm{~mm}$ below the top surface of each sample.

\subsection{Application-specific trapped field profiles}

Based on the results of this investigation, an array of bulk superconductors could be utilized to generate periodic magnetic field profiles of different kinds for specific applications. In magnetic separation, for example, the magnetic force acting on the particles is proportional to the gradient of the magnetic field [61]. Therefore, samples with a high trapped field gradient are preferred in such applications. In contrast, field homogeneity and stability is crucial in applications such as nuclear magnetic resonance (NMR) and magnetic resonance imaging (MRI) [62, 63]. Finally, the magnetisation profile should be such that the distribution of magnetic field in the air gap is as sinusoidal as possible in trapped flux-type electric machines, which requires a different arrangement again of bulk superconductors.

Considering the variety of trapped field profiles required for different HTS applications, the graded technique is potentially important, not only to improve the $\mathrm{Y}-211$ particle distribution throughout the bulk microstructure, but also to allow modification of the traditionally conical shape of the trapped field in bulk superconductors. $J_{c}$ should be enhanced either at the bottom or middle of the sample (Models $B$ and $C$ ) by the graded sample process if a flatter trapped field pattern is required. Finally, improvement of $J_{c}$ in the top region of the sample (Model $D$ ) is required for applications that require a higher trapped field gradient.

The 3D modelling framework presented here provides useful guidance for the graded sample melt processing technique to potentially meet the requirements of a particular application. The distribution, including the size and concentration, of Y-211 in the YBCO bulk precursor powders should be investigated further to achieve these goals. 


\section{Conclusions}

The electromagnetic properties of YBCO bulk superconductors fabricated by top seeded melt growth using a graded technique was studied in this paper. The aim of the graded technique is to provide enhanced homogeneity in the distribution of $\mathrm{Y}-211$ inclusions in the bulk microstructure, thereby overcoming issues related to homogeneity that often arise following melt processing using precursor powders of standard composition. The trapped field was measured for the sample magnetized by the field cooling (FC) process, and a 3D simulation model based on the $\boldsymbol{H}$-formulation was employed to reproduce the experimental results and further study the differences between the samples fabricated by graded and standard TSMG processing with respect to FC and pulsed field magnetization (PFM) techniques. The modelling framework provides a new technique for assessing the performance of various sizes and geometries of graded bulk superconductors, as required for several technologically important applications, and to help guide improvements in processing such samples. Samples with different trapped field profiles can be fabricated specifically for practical bulk superconductor devices based on this concept to provide application-specific trapped field profiles, such as the generation of either a high magnetic field gradient or a high level of uniformity for the traditionally conical, trapped field profile.

\section{Acknowledgements}

Jin Zou and Di Hu would like to acknowledge the support of Churchill College, Cambridge, the China Scholarship Council and the Cambridge Commonwealth, European and International Trust. Dr Mark Ainslie would like to acknowledge the support of a Royal Academy of Engineering Research Fellowship. 


\section{References}

[1] Tomita M and Murakami M 2003 Nature 421 517-20

[2] Durrell J H, Dennis A R, Jaroszynski J, Ainslie M D, Palmer K G B, Shi Y H, Campbell A M, Hull J, Strasik M, Hellstrom E and Cardwell D A 2014 Supercond. Sci. Technol. 27082001

[3] Nariki S, Sakai N and Murakami M 2005 Supercond. Sci. Technol. 18 S126-30

[4] Zhou D F, Mitsuru I, Motohiro M, Brice F, Tetsuya I and Masahiro K 2012 Supercond. Sci. Technol. 25103001

[5] Hull J R 2000 Supercond. Sci. Technol. 13 R1-15

[6] Sino H, Nagashima K and Arai Y 2008 J. Phys.: Conf. Ser. 97012101

[7] Koshizuka N 2006 Physica C 445-448 1103

[8] Werfel F N 2002 Physica C 372-376 1482

[9] Salama K, Selvamanickam V, Gao L and Sun K 1989 Appl. Phys. Lett.54 2352-4

[10] Yang W M et al 1998 Physica C 307 271-6

[11] Cardwell D A 1998 Mater. Sci. Eng. B53 1-10

[12] Murakami M 1990 Mod. Phys. Lett. 4163

[13] Murakami M 1992 Supercond. Sci. Technol. 5 185-203

[14]Devendra Kumar N, Rajasekharan T and Seshubai V 2013 Physica C 495 55-65

[15] Chow J C L, Lo W, Leung H-T, Dewhurst C D and Cardwell D A 1998 Mater. Sci. Eng. B 53 79-85

[16] Endo A, Chauhan H S, Egi T and Shiohara Y 1996 J. Mater. Res. 11795

[17] Endo A, Chauhan H S and Shiohara Y 1996 Physica C 273 107-19

[18] Delamare M P, Walter H, Bringmann B, Leenders A and Freyhardt H C 1999 Physica C 323 10714

[19] Diko P, Todt V R, Miller D J and Goretta K C 1997 Physica C 278 192-200

[20] Zhai W, Shi Y H, Durrell J H, Dennis A R, Rutter N A, Troughton S C, Speller S C and Cardwell D A 2013Supercond. Sci. Technol. 26125021

[21] Pecher R, McCulloch M D, Chapman S J, Prigozhin L and Elliott C M 2003 Proc. 6th EUCAS pp 111

[22] Kajikawa K, Hayashi T, Yoshida R, Iwakuma M and Funaki K 2003 IEEE Trans. Appl. Supercond. 13 3630

[23] Hong Z, Campbell A M and Coombs T A 2006 Supercond. Sci. Technol. 191246 
[24] Brambilla R, Grilli F and Martini L 2007 Supercond. Sci. Technol. 2016

[25] Sirois F, Dione M, Roy F, Grilli F and Dutoit B 2008 J. Phys.: Conf. Ser. 97012030

[26] Ainslie M D, Flack T J, Hong Z and Coombs T A 2011 Int. J. Comput. Math. Electr. Electron. Eng. 30762

[27]Ainslie M D, Rodriguez-Zermeno V M, Hong Z, Yuan W, Flack T J and Coombs T A 2011 Supercond. Sci. Technol.24 045005

[28] Ainslie M D, Flack T J and Campbell A M 2012 Physica C 47250

[29] Zhang M and Coombs T A 2012 Supercond. Sci. Technol.25 015009

[30] Ainslie M D, Yuan W and Flack T J 2013 IEEE Trans. Appl. Supercond.23 4700104

[31] Zermeno V, Grilli F and Sirois F 2013 Supercond. Sci. Technol. 26052001

[32] COMSOL, Inc. http://www.comsol.com/

[33] Withnell T D, Hari-Babu N, Ganney I, Dennis A and Cardwell D A 2008Mat. Sci. Eng. B 151 79-83

[34] Chen D X and Goldfarb R B 1989 J. Appl. Phys.66 2489

[35] Fukai H, Tomita M, Murakami M and Nagatomo T 2002 Supercond. Sci. Technol.15 1054-7

[36] Kim Y B, Hempstead C F and Strnad A R 1963Phys. Rev. Lett.9 306-9

[37] Zhang M, Matsuda K and Coombs T A 2012 Journal of Applied Physics 112043912

[38] Komi Y, Sekino M and Ohsaki H 2009 Physica C.469 1262-5

[39] Shiraishi R and Ohsaki H 2006 IEEE Trans. Appl. Supercond. 16 1794-7

[40] Xu Z 2012 PhD thesis University of Cambridge

[41] Fujishiro H, Naito T, Furuta D, Arayashiki T, Yanagi Y and Itoh Y 2011 Supercond. Sci. Technol. 24 105003

[42] Ogawa J et al 2014 IEEE Trans. Appl. Supercond. 248200304

[43] Xu Z, Lewin R, Campbell A M, Cardwell D A and Jones H 2012 Supercond. Sci. Technol.25 025016

[44] Pathak S K, Yeoh W K, Hari Babu N, Shi Y, lida K, Strasik M and Cardwell D A 2009 Physica C 469 1173

[45] Hari Babu N, Reddy E S, Cardwell D A and Campbell A M 2003 Appl. Phys. Lett.83 4807

[46] Bean C P 1962 Phys. Rev. Lett. 8250

[47] Ainslie M D, Fujishiro H, Ujiie T, Zou J, Dennis A R, Shi Y-H and Cardwell D A 2014 Supercond. Sci. Technol.27065008

[48] Patel A, Glowacki B A 2012 Supercond. Sci. Technol. 25125015 
[49] Fujishiro H and Naito T 2010 Supercond. Sci. Technol.23 105021

[50] Plummer C J G and Evetts J E 1987 IEEE Trans. Magn.23 1179-82

[51] Rhyner J 1993 Physica C 212 292-300

[52] Stavrev S et al2002 IEEE Trans. Magn.38 849-52

[53] Grilli Fet al 2005 IEEE Trans. Appl. Supercond.15 17-25

[54] Ainslie M D 2012 PhD thesis University of Cambridge

[55] Clem J R, Weigand M, Durrell J H and Campbell A M 2011 Supercond. Sci. Technol. 21062002

[56] Campbell A M 2011 Supercond. Sci. Technol.24 091001

[57] Cardwell D A 1998 Mater. Sci. Eng. B53 1

[58] Shi Y, Dennis A R, Xu Z, Campbell A M, Hari-Babu N and Cardwell D A 2010 Supercond. Sci. Technol.23045014

[59] Fujishiro H, Hiyama T,Miura T,Naito T, Nariki S, Sakai N, Hirabayashi I 2009 IEEE Trans. Appl. Supercond.19 3545-8

[60] Yanagi Y, Itoh Y, Yoshikawa M, Oka T, Ikuta H and Mizutani U 2005 Supercond. Sci. Technol.18 839-49

[61] Oka T, Kanayama H, Fukui S, Ogawa J, Sato T, Ooizumi M, Terasawa T, Itoh $Y$ and Yabuno R 2008Physica C4682128-32

[62] Kim SB, Nakano T, Takano R and Hahn S Y 2009 IEEE Trans. Appl. Supercond.19 2273-6

[63] Iwasa Y 2006Physica C445 1088-94 